\title{
AVALIAÇÃO DOS LIMITES RECOMENDADOS DE CARGAS MANUSEADAS NA PRODUÇÃO DE MUDAS EM VIVEIROS FLORESTAIS
}

\author{
Nilton César Fiedler ${ }^{1}$, Fernando Bonelli Wanderley ${ }^{2}$, Pompeu Paes Guimarães ${ }^{3}$, Leonardo Peroni ${ }^{4}$, \\ Rafael Tonetto Alves ${ }^{2}$, Rômulo Môra ${ }^{3}$ \\ ${ }^{1}$ Eng. Florestal, Dr., Depto. de Engenharia Florestal, UFES, Alegre, ES, Brasil - fiedler@pq.cnpq.br \\ ${ }^{2}$ Eng. Florestal, Depto. de Engenharia Florestal, UFES, Alegre, ES, Brasil - fernandobwef@ gmail.com - rafatonetto@ gmail.com \\ ${ }^{3}$ Eng. Florestal, Mestrando em Ciências Florestais, UFES, Alegre, ES, Brasil - pompeupaes@ yahoo.com.br - romulomef@ yahoo.com.br \\ ${ }^{4}$ Graduando em Engenharia Florestal, UFES, Alegre, ES, Brasil - leonardo_peroni2007@ hotmail.com \\ Recebido para publicação: 21/08/2008 - Aceito para publicação: 15/04/2009
}

\begin{abstract}
Resumo
O presente trabalho foi realizado no viveiro de produção de mudas do Centro de Ciências Agrárias da Universidade Federal do Espírito Santo (CCA-UFES), no município de Alegre, sul do estado do Espírito Santo, com o objetivo de analisar as cargas manuseadas por trabalhadores em atividades de produção de mudas de Eucalyptus spp em viveiros florestais e compará-las com os limites recomendados de cargas estabelecidos pelo Instituto Nacional de Segurança e Saúde Ocupacional dos Estados Unidos (NIOSH). Todas as etapas do ciclo de trabalho foram realizadas por métodos manuais. Os valores das cargas manuseadas foram obtidos através de uma balança de precisão. As distâncias horizontais e verticais da carga ao corpo, os ângulos de assimetria e os deslocamentos verticais foram obtidos por medições diretas. As frequências de levantamentos foram analisadas por meio de estudos de tempos e movimentos, utilizando-se o modelo de tempos contínuos, e as dificuldades de manuseio da carga foram avaliadas por meio de fluxograma. De acordo com os resultados obtidos, as mais altas cargas manuseadas pelos trabalhadores ocorreram nas atividades de transporte de substrato, expedição de bandejas e transporte para rustificação das mudas, que ficaram acima dos limites recomendados de cargas, necessitando de cuidados e correções imediatas.
\end{abstract}

Palavras-chave: Ergonomia; viveiro florestal; postura e limites de cargas.

\begin{abstract}
Evaluation of recommended weight handling limits for loads in seedlings production in nursery. This work was developed in nursery seedlings production of the Centro de Ciências Agrárias, Universidade Federal do Espírito Santo (CCA-UFES), Alegre municipality, southern of Espírito Santo State, aiming to analyze purpose loads handled by workers in nurseries activities of Eucalyptus spp seedlings production and to compare them with recommended loads limits established by the National Institute of Occupational Safety and Health in the United States (NIOSH). All stages of the work cycle were done by manual methods. The values of the loads were handled with a precision balance. The horizontal and vertical distances between load to the body, the asymmetry angles and vertical displacements were obtained by direct measurements. The lifting frequencies were analyzed through studies of time and movement, using a time series analysis and handling difficult of evaluated loads throughout flow. According to the results, the highest loads handled by workers occurred in the substrate transport activities, trays dispatch and trays transport to increase of the seedlings resistance, which were above the recommended loads limits, requiring immediate care and corrections.

Keywords: Ergonomics; forest nursery; posture and loads limits.
\end{abstract}

\section{INTRODUÇÃO}

A ergonomia é uma ciência interdisciplinar que compreende a fisiologia e a psicologia do trabalho, objetivando adaptar o posto de trabalho, os instrumentos, as máquinas, os horários e o ambiente às exigências do ser humano, tendo como resultado a melhoria da saúde, do bem-estar, da segurança, do conforto e da produtividade dos funcionários. 
Nas atividades de propagação de plantas, o trabalho realizado envolve várias posturas e manuseio de cargas diferenciadas, podendo ser potencialmente lesivo à saúde dos trabalhadores envolvidos nessas atividades (ALVES et al., 2006).

A postura mais adequada ao trabalhador é aquela que ele escolhe livremente e que pode sofrer variação ao longo do tempo. A concepção dos postos de trabalho ou da tarefa deve favorecer a variação de postura, principalmente a alternância entre as posturas sentada e em pé.

De acordo com Iida (1990), posturas são configurações que um corpo assume ao realizar dada atividade. O registro das posturas corporais adotadas em determinada atividade tem como finalidade principal a identificação de movimentos e/ou posturas que venham a oferecer riscos de lesão ao organismo humano, durante demandas ocupacionais. $\mathrm{Na}$ área florestal, a ocorrência de problemas de lombalgias é muito elevada. Ao manusear uma carga pesada ou ao proceder incorretamente, o trabalhador fica exposto a forças de compressão na união dos segmentos vertebrais $\mathrm{L}_{5}-\mathrm{S}_{1}$, que causam um considerável estresse na região lombar.

As lombalgias não só afetam a saúde do próprio trabalhador como também originam consequências sociais, como o absenteísmo, mudança de profissão por incapacidade laboral e gastos previdenciários, dentre outras, que não devem ser negligenciadas.

$\mathrm{Na}$ avaliação de atividades que exijam elevado esforço físico, deve ser observado o tipo de tarefa com relação ao desgaste físico requerido, considerando o metabolismo, o consumo energético, as pausas, a alimentação, a postura escolhida e o ambiente físico de trabalho. Quando a atividade exigir manuseio de materiais, os principais fatores que interferem são o desgaste energético e as posturas (quando impostas e não variando ao longo do tempo), sendo importante avaliar, a partir do limite recomendado de pesos, se a carga é admissível (WATERS et al., 1993).

Para Amaral (1993), os principais aspectos a serem examinados para resolver os problemas relativos ao levantamento de carga são o processo produtivo (manual ou mecânico), a organização do trabalho (projeto de trabalho, frequência dos levantamentos), o posto de trabalho (posição do peso em relação ao corpo); o tipo da carga (forma, peso, pegas), acessórios de levantamento e o método de trabalho (individual ou coletivo).

Os diferentes modelos biomecânicos contribuem significativamente para a detecção de posturas que possam oferecer riscos de lesão ao organismo, sendo eficientes ferramentas de avaliação de perigo na relação entre o ser humano e a máquina nos mais diversos campos de trabalho, inclusive na área florestal.

Esta pesquisa teve como objetivo analisar as cargas manuseadas por trabalhadores em viveiros de produção de mudas de Eucalyptus spp e compará-las com os limites recomendados quanto à exigência de forças durante as posturas características de cada atividade.

\section{MATERIAL E MÉTODOS}

\section{Região de estudo}

Este trabalho desenvolveu-se no viveiro de produção de mudas do CCA-UFES (Centro de Ciências Agrárias da Universidade Federal do Espírito Santo), no município de Alegre, sul do estado do Espírito Santo, entre agosto de 2007 e julho de 2008. O viveiro produzia mudas de espécies nativas e exóticas com finalidades de pesquisas científicas, doações e vendas para formação de florestas plantadas e recuperação de áreas degradadas. Utilizou-se para esta pesquisa a produção de mudas do gênero Eucalyptus.

\section{População e amostragem}

A população pesquisada era composta por trabalhadores que atuavam nas operações de produção de mudas em viveiros florestais, executadas por métodos manuais (Tabela 1). Todas as etapas do ciclo de trabalho eram realizadas por pessoas de ambos os sexos, sem divisão de tarefas.

O trabalho iniciava com o transporte de substrato do local de armazenagem até o galpão de enchimento de tubetes, onde o substrato era depositado sobre bandejas. Posteriormente, essas bandejas eram deslocadas até a casa de sombra e seguiam-se as atividades de semeadura direta, raleio e mondas. A próxima etapa era a transferência das bandejas para a área de rustificação, realizando-se alternagem e adensamento de tubetes, seleção de mudas e, por último, a atividade de expedição. 
Tabela 1. Atividades desenvolvidas em viveiros de produção de mudas e suas descrições.

Table 1. Activities developed in nurseries seedlings production and its descriptions.

\begin{tabular}{|c|c|}
\hline Atividades & Descrição \\
\hline Transporte de substrato & Carregamento do saco de substrato do galpão até a bancada de enchimento. \\
\hline Deposição de substrato na bandeja & $\begin{array}{l}\text { Colocação do substrato sobre a bandeja que se encontra na bancada de } \\
\text { enchimento. }\end{array}$ \\
\hline Enchimento de tubetes & Preenchimento dos tubetes com substrato. \\
\hline Transporte de bandejas & Carregamento de bandejas da bancada de enchimento até a casa de sombra. \\
\hline Semeadura direta em tubetes & Processo em que as sementes são colocadas nos tubetes. \\
\hline Raleio ou desbaste & Processo que deixa apenas uma muda no recipiente. \\
\hline Mondas & Retirada de plantas invasoras. \\
\hline $\begin{array}{l}\text { Transporte de bandejas para } \\
\text { rustificação das mudas }\end{array}$ & $\begin{array}{l}\text { Carregamento de bandejas da casa de sombra até a área de rustificação, } \\
\text { onde as mudas tornam-se mais resistentes. }\end{array}$ \\
\hline $\begin{array}{l}\text { Aumento do espaçamento entre } \\
\text { tubetes ou alternagem nas bandejas }\end{array}$ & $\begin{array}{l}\text { Mudas são colocadas intercaladamente na bandeja com o objetivo de } \\
\text { redução da competição por luz entre elas, aumentando assim a taxa de } \\
\text { crescimento. }\end{array}$ \\
\hline Adensamento de tubetes na bandeja & Retirada do espaçamento intercalado entre tubetes para expedição. \\
\hline Seleção de mudas & $\begin{array}{l}\text { Separação das mudas por classe de tamanho, objetivando maior } \\
\text { uniformidade para a expedição. }\end{array}$ \\
\hline Expedição & $\begin{array}{l}\text { Transporte das mudas do viveiro para o veículo, tendo elas uma altura } \\
\text { média de } 30 \mathrm{a} 40 \mathrm{~cm} \text {. }\end{array}$ \\
\hline
\end{tabular}

O número mínimo de repetições utilizadas em cada fase deste estudo foi estabelecido com base em uma amostra-piloto, analisada com uso da seguinte fórmula, proposta por Conaw (1977):

$$
n>\left(t^{2} * s^{2}\right) / e^{2}
$$

Em que: $n=$ número de amostras necessárias;

$\mathrm{t}=$ valor tabelado a $5 \%$ de probabilidade (distribuição t de Student);

$\mathrm{s}=$ desvio padrão da amostra;

e $=$ erro admissível a $5 \%$ (média das amostras multiplicada por 0,05 ).

\section{Cálculo do limite recomendado de pesos}

As cargas manuseadas foram medidas com o uso de uma balança de precisão marca Toledo, modelo 9094C/6, com capacidade para até $30 \mathrm{~kg}$.

O limite recomendado de cargas foi avaliado com o uso da equação desenvolvida pelo Instituto Americano de Segurança e Saúde Ocupacional (NIOSH). Verificou-se se as condições do trabalho realizado de levantamento dos pesos eram favoráveis (quando todos os coeficientes dos fatores da equação possuem valor igual a 1) ou desfavoráveis (quando esses coeficientes possuem valor menor que 1). Em condições favoráveis, o limite máximo de manuseio de cargas recomendado pelo NIOSH é de 23 $\mathrm{kg}$ (WATERS et al., 1994).

Em condições desfavoráveis, que ocorreram em todas as etapas do processo, foi aplicada a equação revisada do NIOSH para cada situação, considerando-se os seguintes fatores:

$\mathrm{H}=$ distância horizontal da carga ao corpo, em $\mathrm{cm}$;

$\mathrm{V}=$ distância vertical da carga ao corpo, em cm;

$\mathrm{A}=$ rotação do corpo ou ângulo assimétrico, em graus;

$\mathrm{D}=$ deslocamento vertical da carga, em $\mathrm{cm}$;

$\mathrm{F}=$ frequência de levantamentos por minuto;

$\mathrm{C}=$ dificuldade de manuseio da carga.

Todos os dados foram levantados por medições diretas, com exceção da frequência de levantamentos e da dificuldade de manuseio da carga. O valor da frequência foi analisado por meio de estudos de tempos e movimentos, utilizando-se o método de tempos contínuos. A dificuldade de manuseio de carga foi analisada por meio de um fluxograma de decisão.

Com os valores médios de cada item da equação do NIOSH, foram calculados os coeficientes que variam de zero, indicando uma situação mais desfavorável, a um, indicando uma melhor condição. 
Nessa equação, supõe-se que o trabalhador escolha sua postura e segure a carga com as duas mãos. A carga máxima de $23 \mathrm{~kg}$ é multiplicada por 6 (seis) coeficientes, sendo os limites recomendados de pesos obtidos pela seguinte equação:

$$
L P R=L C * H M * V M * D M * A M * F M * C M
$$

Em que: $L P R=$ limite recomendado de peso $(\mathrm{kg})$;

$L C=$ constante de carga $(23 \mathrm{~kg})$;

$H M=$ coeficiente de distância horizontal;

$V M=$ coeficiente de distância vertical;

$D M=$ coeficiente de deslocamento vertical;

$A M=$ coeficiente assimétrico;

$F M=$ coeficiente de frequência de levantamentos;

$C M=$ coeficiente de manuseio da carga.

O levantamento de uma carga igual ao valor da constante de carga $(23 \mathrm{~kg})$ em condições ideais seria realizado por $75 \%$ da população feminina e por $90 \%$ da masculina, de maneira tal que a força de compressão no disco L5-S1, produzida pelo levantamento, não supere os 3426,3 N (Newton) (WATERS et al., 1994). A $L C$ é a carga máxima recomendada para um levantamento em condições ótimas.

O coeficiente de distância horizontal é a distância entre o ponto médio das pegas na carga e do ponto médio entre os tornozelos. Assim, a distância de 0 a $25 \mathrm{~cm}$ fornece um coeficiente igual a 1 . Em distâncias superiores a $63 \mathrm{~cm}$, ele será igual a zero. O cálculo do coeficiente para as demais distâncias horizontais é obtido através da seguinte equação:

$$
H M=25 / H
$$

Em que: $H M=$ coeficiente de distância horizontal da carga ao corpo;

$H$ = distância horizontal da carga ao corpo, em cm.

O coeficiente de distância vertical mede a distância do piso até o ponto de pega na carga. Fornece valores maiores entre 70 e $80 \mathrm{~cm}$ de altura e, quando a carga estiver no chão, o coeficiente a ser multiplicado é 0,78 . Acima de $175 \mathrm{~cm}$, o coeficiente é zero, sendo inviável a execução do trabalho nessas condições. O coeficiente é calculado através da seguinte equação para as demais distâncias:

$$
V M=(1-0,003 *|V-75|)
$$

Em que: $V M=$ coeficiente vertical;

$V=$ distância vertical da carga ao corpo, em $\mathrm{cm}$.

O coeficiente de deslocamento vertical refere-se à diferença entre a altura inicial e final da carga, sendo ótimo em valores iguais ou inferiores a $25 \mathrm{~cm}$ de deslocamento, com coeficiente igual a 1 . Acima de $175 \mathrm{~cm}$, ele assume valor igual a zero, indicando mudanças imediatas no trabalho. De 25 a $175 \mathrm{~cm}$, o coeficiente é calculado usando a seguinte equação:

$$
D M=(0,82+4,5 / D)
$$

Em que: $D M=$ coeficiente de deslocamento vertical;

$D=$ deslocamento vertical da carga, em $\mathrm{cm}$.

O componente assimétrico refere-se ao ângulo de giro do corpo do trabalhador no plano sagital, podendo assumir valores de coeficientes mais próximos de 1 quanto menor for esse ângulo. Se não houver nenhum giro do corpo durante o levantamento da carga, o coeficiente será igual a 1 . Acima de $135^{\circ}$ de giro, ele é zero, ficando inviável a continuação do trabalho dessa forma. Entre 15 a $135^{\circ}$, o coeficiente varia de acordo com a seguinte equação: 


$$
A M=\left(1-0,0032 * A^{\circ}\right)
$$

Em que: $A M=$ coeficiente assimétrico;

$A$ = ângulo assimétrico, em graus.

O componente de frequência de levantamentos por minuto $(F M)$ varia segundo o número de levantamentos por minuto, a duração da jornada de trabalho executando esse tipo de tarefa e a altura vertical da carga ao corpo $(V)$, conforme mostrado na tabela 2. Essa frequência é medida em intervalos médios a cada 15 minutos de observação.

Tabela 2. Coeficiente de frequência de levantamentos.

Table 2. Coefficient of lifting frequency.

\begin{tabular}{|c|c|c|c|c|c|c|}
\hline \multirow{3}{*}{$\begin{array}{l}\text { Número de levantamentos } \\
\text { por minuto }\end{array}$} & \multicolumn{6}{|c|}{ Duração do trabalho } \\
\hline & \multicolumn{2}{|c|}{$\leq 1$ hora } & \multicolumn{2}{|c|}{ Entre 1 e 2 horas } & \multicolumn{2}{|c|}{ Entre 2 e 8 horas } \\
\hline & $\begin{array}{c}\mathrm{V}<76,2 \\
\quad(\mathrm{~cm})\end{array}$ & $\begin{array}{c}V \geq 76,2 \\
(\mathrm{~cm})\end{array}$ & $\begin{array}{c}\mathrm{V}<76,2 \\
\quad(\mathrm{~cm})\end{array}$ & $\begin{array}{c}V \geq 76,2 \\
(\mathrm{~cm})\end{array}$ & $\begin{array}{c}\mathrm{V}<76,2 \\
\quad(\mathrm{~cm})\end{array}$ & $\begin{array}{c}V \geq 76,2 \\
(\mathrm{~cm})\end{array}$ \\
\hline$\leq 0,2$ & 1,00 & 1,00 & 0,95 & 0,95 & 0,85 & 0,85 \\
\hline 0,5 & 0,97 & 0,97 & 0,92 & 0,92 & 0,81 & 0,81 \\
\hline 1 & 0,94 & 0,94 & 0,88 & 0,88 & 0,75 & 0,75 \\
\hline 2 & 0,91 & 0,91 & 0,84 & 0,84 & 0,65 & 0,65 \\
\hline 3 & 0,88 & 0,88 & 0,79 & 0,79 & 0,55 & 0,55 \\
\hline 4 & 0,84 & 0,84 & 0,72 & 0,72 & 0,45 & 0,45 \\
\hline 5 & 0,80 & 0,80 & 0,60 & 0,60 & 0,35 & 0,35 \\
\hline 6 & 0,75 & 0,75 & 0,50 & 0,50 & 0,27 & 0,27 \\
\hline 7 & 0,70 & 0,70 & 0,42 & 0,42 & 0,22 & 0,22 \\
\hline 8 & 0,60 & 0,60 & 0,35 & 0,35 & 0,18 & 0,18 \\
\hline 9 & 0,52 & 0,52 & 0,30 & 0,30 & 0,00 & 0,15 \\
\hline 10 & 0,45 & 0,45 & 0,26 & 0,26 & 0,00 & 0,13 \\
\hline 11 & 0,41 & 0,41 & 0,00 & 0,23 & 0,00 & 0,00 \\
\hline 12 & 0,37 & 0,37 & 0,00 & 0,21 & 0,00 & 0,00 \\
\hline 13 & 0,00 & 0,34 & 0,00 & 0,00 & 0,00 & 0,00 \\
\hline 14 & 0,00 & 0,31 & 0,00 & 0,00 & 0,00 & 0,00 \\
\hline 15 & 0,00 & 0,28 & 0,00 & 0,00 & 0,00 & 0,00 \\
\hline$>15$ & 0,00 & 0,00 & 0,00 & 0,00 & 0,00 & 0,00 \\
\hline
\end{tabular}

Para estabelecer o tipo de encaixe, tem-se uma árvore de decisão, conforme mostrado na figura 1. De acordo com as características do objeto (apresenta recipiente ou está solto, volumoso ou não), do ângulo de flexão dos dedos e da condição do manuseio, encontra-se o tipo de encaixe das mãos na carga, que pode ser bom, médio ou ruim.

\begin{tabular}{|c|c|c|c|c|}
\hline COM RECIPIENTE & & & & OBJETO SOLTO \\
\hline $\operatorname{Sim} \downarrow$ & & & & $\operatorname{sim} \downarrow$ \\
\hline Recipiente ótimo? & $\begin{array}{l}\rightarrow \\
\text { Não }\end{array}$ & RUIM & $\begin{array}{l}\leftarrow \\
\operatorname{sim}\end{array}$ & Objeto volumoso? \\
\hline $\operatorname{Sim} \Downarrow$ & & não $\uparrow$ & & não $\downarrow$ \\
\hline Manuseio ótimo? & $\begin{array}{c}\rightarrow \\
\text { Não }\end{array}$ & Dedos flexionados a $90^{\circ} ?$ & $\begin{array}{c}\leftarrow \\
\text { não }\end{array}$ & Pega ótima? \\
\hline $\operatorname{Sim} \downarrow$ & & & & $\operatorname{sim} \downarrow$ \\
\hline ВОМ & & & & BOM \\
\hline
\end{tabular}

Figura 1. Fluxo de decisão para o tipo de encaixe das mãos na carga.

Figure 1. Decision flow for the plug hands type in charge. 
O coeficiente de manuseio da carga (CM) varia de acordo com a distância vertical do encaixe ao piso e do tipo de encaixe, tendo coeficientes de valor igual a 1 no encaixe bom e 0,90 no encaixe ruim (Tabela 3).

Tabela 3. Coeficiente de manuseio da carga de acordo com o tipo de encaixe das mãos.

Table 3. Load hand lifting coefficient according to hand socket.

\begin{tabular}{lcc}
\hline \multicolumn{3}{c}{ Coeficiente de manuseio $(\mathbf{C M})$} \\
\hline Tipo de encaixe & $\mathbf{V}<\mathbf{7 5}(\mathbf{c m})$ & $\mathbf{V} \geq \mathbf{7 5}(\mathbf{c m})$ \\
\hline Bom & 1,00 & 1,00 \\
Médio & 0,95 & 1,00 \\
Ruim & 0,90 & 0,90 \\
\hline
\end{tabular}

O índice de levantamento (IL) significa a estimativa do nível de estresse no trabalho, realizada de acordo com o NIOSH, definido pela relação entre o peso médio da carga levantada e o limite de peso recomendado $(L P R)$, ou seja:

Em que: $P M=$ peso manuseado, em $\mathrm{kg}$;

$$
I L=P M / L P R
$$

$L P R=$ limite recomendado de pesos, em $\mathrm{kg}$.

Se os valores do índice forem abaixo de 0,9 , o trabalho é sempre seguro. Valores entre 0,9 e 1,2 também são considerados seguros, a menos que a força de trabalho seja predominantemente de pessoas do sexo feminino e de baixa capacidade de força física. Entre 1,2 e 2,0, deve-se tomar cuidado quanto às condições de trabalho. Acima de 2,0, deve-se direcionar forte atenção às condições de trabalho.

\section{Procedimento estatístico}

Os resultados obtidos através do limite de pesos recomendados foram submetidos a uma análise de variância no delineamento inteiramente casualizado. As médias das máquinas foram comparadas pelo teste de Tukey, a 5\% de probabilidade, apresentando diferenças estatísticas significativas.

\section{RESULTADOS E DISCUSSÃO}

Para obter um índice de confiança de 95\%, o número mínimo de amostras necessárias foi, em média, de 31 observações, mas foram feitas 143 medições das posturas típicas dos trabalhadores por atividade (média). O desvio padrão variou de zero (nas atividades onde o LPR foi nulo) a 1,56, dependendo da atividade avaliada.

As atividades nos viveiros de produção de mudas encontram-se numeradas na tabela 4. Na tabela 5 estão relacionados, para cada tipo de atividade do ciclo de trabalho, a distância horizontal média $(\mathrm{H})$, em cm; o coeficiente horizontal (HM); a distância vertical média (V), em cm; o coeficiente vertical (VM); o deslocamento vertical médio (D), em cm; o coeficiente de deslocamento (DM); o ângulo assimétrico médio (A), em graus; o coeficiente assimétrico (AM); a frequência média de levantamentos (F); o coeficiente de frequência (FM); o tipo de encaixe das mãos (C); o coeficiente de encaixe (CM); o peso médio da carga, em kg; o limite de peso recomendado (LPR), em kg; o índice de levantamento (IL); e o número de mãos utilizadas em cada atividade pelos trabalhadores.

As menores cargas manuseadas pelos trabalhadores, de acordo com a tabela 5, foram registradas nas atividades de monda, semeadura direta, seleção de mudas, raleio, adensamento e alternagem de tubetes, com carga igual ou inferior a $0,3 \mathrm{~kg}$.

A atividade de transporte de substrato apresentou o maior valor médio de manuseio de carga $(24,8 \mathrm{~kg})$, seguida pela expedição de bandejas $(11,04 \mathrm{~kg})$, transporte de bandejas para o local de rustificação das mudas $(10,77 \mathrm{~kg})$ e transporte de bandejas com substrato $(10,53 \mathrm{~kg})$, ficando estas atividades acima do limite de peso recomendado e com índice de levantamento entre 1,15 e 1,53.

Pela classificação do método, deve-se tomar cuidado com as condições de trabalho nessas atividades, mesmo para o transporte de bandejas com substrato, que apresentou índice de levantamento de 1,1 , porque pessoas do sexo feminino e de baixa capacidade de força física participam dessa etapa de trabalho. 
Tabela 4. Atividades do ciclo de trabalho em viveiros de produção de mudas e suas respectivas numerações para a equação do NIOSH.

Table 4. Work stages activities on forest nurseries and their numbering to NIOSH equation.

\begin{tabular}{ll}
\hline $\mathbf{N}^{\mathbf{o}}$ & \multicolumn{1}{c}{ Atividade do ciclo de trabalho } \\
\hline 1 & Transporte de substrato \\
2 & Colocação de substrato em bandejas \\
3 & Enchimento de tubetes \\
4 & Transporte de bandejas com substrato \\
5 & Semeadura direta em tubetes \\
6 & Raleio tubete \\
7 & Mondas tubete \\
8 & Transporte para rustificação das mudas em bandejas \\
9 & Aumento do espaçamento nas bandejas \\
10 & Adensamento nas bandejas \\
11 & Seleção de mudas \\
12 & Expedição de bandejas \\
\hline
\end{tabular}

Tabela 5. Aplicação da equação revisada do NIOSH sobre as diferentes atividades de produção de mudas, para obtenção dos limites recomendados de pesos (LPR) e do índice de levantamento (IL).

Table 5. NIOSH revised equation implemented on various activities of seedlings production to obtain the weight limits recommended (LPR) and lifting index (IL).

\begin{tabular}{lcccccccccccccccc}
\hline Atividade & $\mathbf{H}$ & $\mathbf{H M}$ & $\mathbf{V}$ & $\mathbf{V M}$ & $\mathbf{D}$ & $\mathbf{D M}$ & $\mathbf{A}$ & $\mathbf{A M}$ & $\mathbf{F}$ & $\mathbf{F M}$ & $\mathbf{C}$ & $\mathbf{C M}$ & $\mathbf{L P R}$ & Peso & IL & $\begin{array}{c}\mathbf{N}^{\mathbf{0}} \\
\text { mãos }\end{array}$ \\
\hline 1 & 31.83 & 0.78 & 88.67 & 0.93 & 0 & 1 & 0 & 1 & 0.5 & 0.97 & Médio & 1 & 16.18 & 24.8 & 1.53 & Duas \\
2 & 34.50 & 0.72 & 116.13 & 0.87 & 90.00 & 0.87 & 0 & 1 & 0.5 & 0.97 & Bom & 1 & 12.16 & 4.73 & 0.39 & Uma \\
3 & 34.50 & 0.72 & 116.13 & 0.87 & 0 & 1 & 0 & 1 & 2 & 0.91 & Bom & 1 & 13.11 & 4.73 & 0.36 & Uma \\
4 & 38.67 & 0.65 & 111.7 & 0.89 & 21.67 & 1 & 90 & 0.71 & 0.5 & 0.97 & Médio & 1 & 9.16 & 10.53 & 1.15 & Duas \\
5 & 56.90 & 0.44 & 105.00 & 0.91 & 0 & 1 & 0 & 1 & $>15$ & 0 & Bom & 1 & 0.00 & 0.3 & - & Uma \\
6 & 32.28 & 0.77 & 101.00 & 0.92 & 101.00 & 0.86 & 0 & 1 & 5 & 0.80 & Bom & 1 & 11.21 & 0.125 & 0.01 & Duas \\
7 & 33.44 & 0.75 & 92.86 & 0.95 & 92.86 & 0.87 & 0 & 1 & 12 & 0.37 & Bom & 1 & 5.28 & 0.01 & 0.00 & Uma \\
8 & 39.15 & 0.64 & 118.62 & 0.87 & 12.38 & 1 & 90 & 0.71 & 0.5 & 0.97 & Médio & 1 & 8.82 & 10.77 & 1.22 & Duas \\
9 & 40.23 & 0.63 & 77.00 & 0.99 & 0 & 1 & 0 & 1 & $>15$ & 0 & Bom & 1 & 0.00 & 0.125 & - & Uma \\
10 & 39.68 & 0.63 & 88.21 & 0.96 & 8.09 & 1 & 0 & 1 & $>15$ & 0 & Bom & 1 & 0.00 & 0.125 & - & Uma \\
11 & 45.44 & 0.55 & 105.00 & 0.91 & 0 & 1 & 0 & 1 & $>15$ & 0 & Bom & 1 & 0.00 & 0.25 & - & Duas \\
12 & 39.52 & 0.63 & 119.22 & 0.87 & 30.00 & 0.97 & 90 & 0.71 & 2 & 0.91 & Bom & 1 & 7.90 & 11.04 & 1.40 & Duas \\
\hline
\end{tabular}

H: distância horizontal média; HM: coeficiente horizontal; V: distância vertical média; VM: coeficiente vertical; D: deslocamento vertical médio; DM: coeficiente de deslocamento; A: ângulo assimétrico médio; AM: coeficiente assimétrico; F: frequência média de levantamentos; FM: coeficiente de frequência; C: tipo de encaixe das mãos; CM: coeficiente de encaixe; Peso: peso médio da carga; LPR: limite de peso recomendado; IL: índice de levantamento.

Como forma de intervir nessas atividades, deve-se analisar os coeficientes que mais contribuíram para a redução dos limites recomendados de cargas, que nesse caso foram o ângulo de giro com a carga e a distância horizontal da carga ao corpo, propondo mudanças da forma de trabalho através de cursos de treinamento e de reciclagem. Além dessas correções, o transporte de substrato deve ser feito por um carrinho de distribuição ou, ainda, ter seu peso diminuído, para que, num levantamento sob condições ótimas, a força de compressão no disco L5-S1 da coluna vertebral, produzida no levantamento, não supere os $3426,3 \mathrm{~N}$.

Segundo Dul; Weerdmeester (1995), o manuseio de cargas com pesos acima dos limites permissíveis por longos períodos pode comprometer seriamente as articulações. As atividades em viveiros florestais exigem movimentos repetitivos e a permanência prolongada de posturas em pé e inadequadas, fatores estes determinantes para o aparecimento de doenças osteomusculares relacionadas ao trabalho (DORT). 
Devido à elevada frequência de levantamentos por minuto (maior que 15), as atividades de semeadura direta, alternagem e adensamento de tubetes e seleção de mudas apresentaram, durante a aplicação da equação revisada do NIOSH, um coeficiente igual a zero e, consequentemente, um limite recomendado de cargas igual a zero, inviabilizando a realização da operação da forma que é executada.

A simples opção pela sugestão de redução da frequência de levantamentos poderá acarretar uma diminuição no rendimento da atividade e, consequentemente, aumento nos custos totais, o que, talvez, não seja viável técnica e economicamente. Devem ser adotadas medidas, através de uma avaliação da carga física de trabalho, que beneficiem a saúde do trabalhador, como aquisição de carrinhos de distribuição adequados ao tipo de trabalho florestal, diminuindo posições incômodas, e a ocorrência de pausas no decorrer do dia. Segundo Alves et al. (2000), em estudos ergonômicos, medem-se os índices fisiológicos com o objetivo de se determinar o limite da atividade física que um indivíduo pode exercer, sendo possível determinar a duração da jornada de trabalho e a duração e frequência de pausas, para que o trabalho não traga desgastes excessivos durante a execução da atividade.

Outros procedimentos podem ser adotados para reduzir os esforços excessivos sobre a coluna vertebral e melhorar as condições de trabalho, tais como promover palestras sobre como adotar posturas corretas no levantamento e transporte de materiais (manter a coluna reta e usar a musculatura das pernas, manter a carga o mais próximo possível do corpo, procurar manter cargas simétricas usando as duas mãos, para evitar a criação de movimentos em torno do corpo) e incentivar a preservação da saúde do trabalhador.

As atividades de colocação de substrato em bandejas, enchimento de tubetes, semeadura direta, mondas e o adensamento e aumento do espaçamento em bandejas apresentaram situações de levantamento de carga com uma das mãos. O NIOSH recomenda que seja feita uma ponderação quanto à consideração de risco, ou seja, se o cálculo do índice de levantamento tiver mostrado um determinado valor (seguro, seguro com restrições, risco ou alto risco), considerar um nível acima. Portanto, o índice dessas atividades realizadas com uma das mãos alcançou valores entre 0,9 e 1,2, sendo considerados também seguros, com restrições para a força de trabalho de pessoas do sexo feminino e de baixa capacidade de força física.

Devido às atividades de viveiros de produção de mudas trabalharem, em grande parte, com cargas manuseadas de baixo peso e elevada frequência de levantamentos, torna-se necessária a aplicação de outros modelos biomecânicos na detecção de posturas lesivas ao organismo, na medida em que são introduzidas outras variáveis na análise desses modelos, contribuindo para a melhoria das condições de vida dos trabalhadores e consequentemente para o aumento da produtividade. A figura 2 compara as diferenças entre os pesos manuseados e os limites de peso recomendados para os trabalhadores.

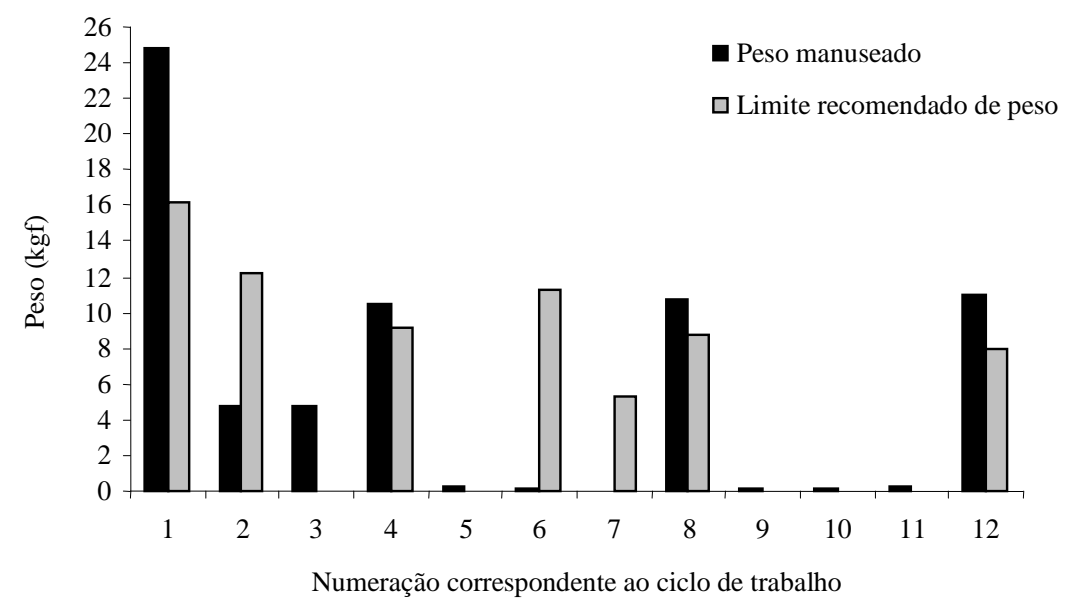

Figura 2. Peso manuseado e limite recomendado de peso para os trabalhadores em viveiros florestais. Figure 2. Weight handled and weight limit recommended for workers in forest nurseries. 
Os resultados da análise de variância para o delineamento inteiramente casualizado do limite recomendado de cargas são apresentados na tabela 6 . Foram verificadas diferenças significativas ao nível de $5 \%$ de probabilidade pelo teste $\mathrm{F}$, sendo então necessária a realização do teste de Tukey $(\mathrm{p}=0,05)$, para verificar diferenças entre as atividades.

Tabela 6. Resultados da análise de variância para o limite recomendado de peso.

Table 6. Results of variance analysis for weight limits recommended.

\begin{tabular}{lcccc}
\hline FV & GL & SQ & QM & F cal \\
\hline Atividade & 11 & 21342,23 & 1940,20 & $1888,22^{*}$ \\
Resíduo & 1439 & 1478,61 & 1,03 & \\
Total & 1450 & 22820,84 & 1941,23 & \\
\hline
\end{tabular}

* Significativo a 5\% de probabilidade.

De acordo com os resultados apresentados na tabela 6 , foi necessária a utilização de um teste de comparações de médias para avaliação das similaridades e das diferenças existentes entre as atividades. Os resultados do teste de Tukey para o nível do limite recomendado de cargas nas atividades são apresentados na tabela 7 .

O teste de comparações de médias mostra diferenças estatísticas entre as atividades do ciclo de trabalho comumente desenvolvido em um viveiro florestal e também verifica que os limites recomendados de cargas podem não diferir estatisticamente entre si, como no caso das atividades de seleção de mudas, enchimento de tubetes e transporte de bandejas com substrato, e das atividades de adensamento nas bandejas, expedição de bandejas e semeadura direta em tubetes, além dos tratamentos que obtiveram médias zero. Todas as outras atividades (raleio e mondas em tubetes) diferiram estatisticamente das demais.

Tabela 7. Resultados do teste de comparações de médias de Tukey para o limite recomendado de cargas.

Table 7. Results from Tukey test of comparison between averages for weight limits recommended.

\begin{tabular}{lc}
\hline Tratamento & Médias \\
\hline Seleção de mudas & $14.477 \mathrm{~A}$ \\
Enchimento de tubetes & $14.228 \mathrm{~A}$ \\
Transporte de bandejas com substrato & $13.348 \mathrm{~A}$ \\
Mondas tubete & $11.105 \mathrm{~B}$ \\
Adensamento nas bandejas & $9.053 \mathrm{C}$ \\
Expedição de bandejas & $8.532 \mathrm{C}$ \\
Semeadura direta em tubetes & $8.203 \mathrm{C}$ \\
Raleio tubetes & $1.281 \mathrm{D}$ \\
Transporte de substrato & $0.000 \mathrm{E}$ \\
Colocação de substrato em bandejas & $0.000 \mathrm{E}$ \\
Transporte para rustificação das mudas & $0.000 \mathrm{E}$ \\
Aumento espaçamento nas bandejas & $0.000 \mathrm{E}$ \\
\hline
\end{tabular}

De acordo com a tabela 7, as atividades que apresentaram as maiores médias para os limites recomendados de cargas foram a seleção de mudas, o enchimento de tubetes e o transporte de bandejas com substrato, e o menores limites recomendados de cargas foram constatados nos tratamentos que apresentaram média, zero conforme tabela 7. Isso demonstra que essas atividades são as que mais precisam de mudanças, de modo a diminuir o LPR, ao contrário dos tratamentos que apresentaram valor de LPR igual a zero, ou seja, são atividades que já apresentam o LPR adequado para as atividades. As outras atividades descritas necessitam de pequenos ajustes, de modo a estabelecer um maior no conforto para od trabalhadores que realizam essas atividades.

As atividades que demandaram LPR intermediários não causam tanto desconforto quanto as atividades citadas acima e, por isso, precisam apenas de intervenções preventivas que possam minimizar seus impactos ao longo do tempo. As etapas com LPR de média "zero" são atividades ideais e devem ser seguidas para que a produtividade do viveiro seja contínua e sustentável.

\section{CONCLUSÕES}

De acordo com os resultados obtidos, concluiu-se que:

- A atividade que exigiu as posturas mais inadequadas ergonomicamente durante todo o ciclo de trabalho foi o transporte de substrato. 
- As atividades de semeadura direta, aumento do espaçamento entre mudas, adensamento de tubetes e seleção de mudas apresentaram, em função do elevado número de repetições por minuto, coeficientes iguais a zero e, consequentemente, limites recomendados de cargas iguais a zero, inviabilizando a realização da operação da forma como é executada.

- As atividades de transporte de substrato, transporte de bandejas com substrato, expedição de bandejas e transporte para rustificação de mudas ultrapassaram os limites de cargas recomendados, além de registrarem índices de levantamento elevados, necessitando de correção imediata.

- As situações em que as cargas manuseadas estavam menos prejudiciais para os trabalhadores ocorreram nas atividades de monda e raleio, com manuseio de carga muito menor que o limite permitido, podendo ser realizados sem nenhuma correção.

- Para aumentar o limite recomendado de cargas nas atividades do ciclo de trabalho, deve-se atuar na otimização dos coeficientes. Um exemplo que tem alcançado bons resultados é atuar na melhoria das posturas adotadas durante o trabalho, que deve ser feita com a introdução de cursos de treinamento e reciclagem.

- Outras recomendações que beneficiam a saúde do trabalhador são a introdução de pausas no decorrer do horário de trabalho e a aquisição de carrinhos de distribuição adequados ao tipo de trabalho florestal.

- Aplicação de outros modelos biomecânicos na detecção de posturas que possam oferecer riscos de lesão ao organismo, devido à ocorrência, em grande parte, de cargas manuseadas com baixo peso e elevada frequência de levantamentos por minuto.

\section{REFERÊNCIAS}

ALVES, J. U.; SOUZA, A. P. de; MINETTE, L. J.; GOMES, J. M.; SILVA, K. R. da; MARÇAL, M. A.; SILVA, E. P. da. Avaliação biomecânica de atividades de produção de mudas de Eucalyptus ssp. Revista Árvore, Viçosa-MG, v. 30, n. 3, p. 331-335, 2006.

ALVES, J. U.; SOUZA, A. P.; MINETTI, L. J.; GOMES, J. M. Avaliação da carga de trabalho físico de trabalhadores que atuam na atividade de propagação de Eucaliptus spp. In: SIMPÓSIO BRASILEIRO SOBRE ERGONOMIA E SEGURANÇA DO TRABALHO FLORESTAL E AGRÍCOLA, 1, 2000, Belo Horizonte, MG. Anais do... Belo Horizonte: Ergoflor, 2000. p. 129 - 134.

AMARAL, F. G. O método NIOSH: método pratico para avaliar cargas e o risco dorso-lombar associado. In: CONGRESSO LATINO-AMERICANO E SEMINÁRIO BRASILEIRO DE ERGONOMIA 2.; 6:1993: Florianópolis. Anais... Rio de Janeiro: ALBERGO; Brasília, DF: FUNDACENTRO, p. 240-247, 1993.

CONAW, P. L. Estatística. São Paulo: E. Blucher. 1977. 264 p.

DUL, J.; WEERDMEESTER, B. Ergonomia prática. São Paulo: E. Blucher 1995. 147 p.

IIDA, I. Ergonomia: projeto e produção. São Paulo: E. Blucher, 1990. 465 p.

WATERS, T. R.; ANDERSON, V. P.; GARG, A. Revised NIOSH equation for the design and evaluation of manual lifting tasks. Ergonomics, Abingdon, v. 36, n. 7, p. 749-776, 1993.

WATERS, T. R.; ANDERSON, V. P.; GARG, A. Applications manual for the revised NIOSH lifting equation. Cincinnati: U. S. Department of Health Human Services, 1994. 119 p. 